\title{
Embedded Sensor Networks
}

\author{
Sitharama S. Iyengar \\ Roy Paul Daniels Professor and Chair \\ Sensor Network Laboratory - Dept. of Computer Science \\ Louisiana State University, USA
}

\begin{abstract}
Embedded sensor networks are distributed systems for sensing and in situ processing of spatially and temporally dense data from resource-limited and harsh environments such as seismic zones, ecological contamination sites are battle fields. From an application point of view, many interesting questions arise from sensor network technology that go far beyond the networking/computing aspects of the embedded system. This talk presents an overview of various open problems that are both of mathematical and engineering interests. These problems include sensor-centric quality of routing/energy optimization among other graph theoretic problems.
\end{abstract}

Bio: Dr. S. S. Iyengar is the Chairman and Roy Paul Daniels Distinguished Professor of Computer Science at Louisiana State University and is also Satish Dhawan Chaired Professor at Indian Institute of Science. He has been involved with research in high-performance algorithms, data structures, sensor fusion, data mining, and intelligent systems since receiving his Ph.D. degree in 1974. He has directed over $40 \mathrm{Ph}$.D. students, many of whom are faculty at major universities worldwide or scientists or engineers at national labs/industry around the world. His publications include 15 books (authored or coauthored, edited; Prentice-Hall, CRC Press, IEEE Computer Society Press, John Wiley \& Sons, etc.) and over 350 research papers in refereed journals and conferences. He has won many best paper awards from various conferences. His research has been funded by NSF, DARPA, DOE-ORNL, ONR among others. He is a Fellow of ACM, Fellow of the IEEE, Fellow of AAAS. Dr. Iyengar is the winner of the many IEEE Computer Society Awards. Dr. Iyengar was awarded the LSU Distinguished Faculty Award for Excellence in Research, the Hub Cotton Award for Faculty Excellence, and many other awards at LSU. He received the Prestigious Distinguished Alumnus Award from Indian Institute of Science, Bangalore in 2003. Also, Elected Member of European Academy of Sciences (2002). He is a member of the New York Academy of Sciences. He has been the Program Chairman for many national/international conferences. He has given over 100 plenary talks and keynote lectures at numerous national and international conferences. 